FZMw Jg. 1 (1998) H. 1, S. 51-61

- [B] Krebs, Wolfgang: Humanismus und Motette im 15. und 16. Jahrhundert (Internationale Tagung, Münster, 2.-4. Februar 1998)

○ [B] Eichhorn, Andreas: Musik und Wissenschaft im Dialog. Eine Diskussionsveranstaltung des Musikwissenschaftlichen Instituts Frankfurt/Main im Rahmen von Wissenschaft erleben - Das Uni-Universum

○ [I] : Der Komponist Dr. Karlheinz Essl

○ [I] : Deutschsprachige Mailinglisten

$\circ[\mathrm{I}]$ : Verzeichnis von Zeitschriften - Index von Komponisten

$\circ[\mathrm{I}]$ : Electronic Musicological Review

- $\underline{\text { Die Autoren }}$

\title{
BERICHTE
}

\section{Humanismus und Motette im 15. und 16. Jahrhundert (Internationale Tagung, Münster, 2.-4. Februar 1998)}

von Wolfgang Krebs

Die Erkenntnis, daß sich humanistische Grundströmungen als geistige Haltungen und die Musik des 15. und 16. Jahrhunderts (entgegen früherer Anschauungen, die eher noch von einem mehr oder weniger beziehungslosen parallelen Verlauf ausgingen) in vielfältiger Weise berühren, ist als solche nicht mehr ganz neu. Doch die Diskussion über den musikalischen Humanismus wurde zuweilen unter vereinseitigenden Vorzeichen geführt. Nicht, daß der Tradition der Humanistenode, den Tendenzen zur Einstimmigkeit oder den Bestrebungen der Rezeption antiker Rhetorik nebst deren Übertragung auf die Musik die Relevanz für die Umgrenzung humanistischer Haltungen zu bestreiten wäre. Doch dürften die Einflüsse des Humanismus andererseits auch in die mehrstimmige Kunstmusik hineinreichen, mithin im besonderen die Gattung der Motette betreffen. Indes, zur Zeit läßt sich ein Mißverhältnis zwischen der mittlerweile durchaus sehr erfolgreichen Erschließung musikalischer Quellen auf der einen, den 
Unzulänglichkeiten der interpretatorischen Ansätze auf der anderen Seite kaum leugnen.

Die Internationale Tagung Humanismus und Motette im 15. und 16. Jahrhundert wurde vom Musikwissenschaftlichen Seminar der Westfälischen Wilhelms-Universität Münster unter der Leitung von Prof. Dr. Klaus Hortschansky organisiert und aus Mitteln der Deutschen Forschungsgemeinschaft gefördert. Sie fand im Zentrum für NiederlandeStudien, Münster, statt. Die Tagung versuchte, anhand einiger vorab formulierter Leitlinien (die dennoch nicht schon den Anspruch erhoben, endgültige Resultate oder Interpretationsmuster zu sein) einzelne Diskussionsfelder zu benennen. Dazu rechneten die Rolle der Auftraggeber und das Mäzenatentum, die Spezifika der Textformen des 15. und 16. Jahrhunderts, Fragen der Symbolik, das Verhältnis von Wort und Ton vor dem Hintergrund des Deklamationsproblems, die Bedeutung des durchimitierten Satzes, die Auswirkungen der Neubelebung aristotelischer Poetik und antiker Ethoslehre oder die Divergenzen und Übereinstimmungen mittelalterlicher und renaissancehumanistischer Rezeption antiker Musiktheorie. Die Referate differenzierten anhand besonderer Einzel-

fragen weiter. Der Vortrag über das Distichon in Motetten des deutschsprachigen Raumes im 16. Jahrhundert (Prof. Dr. Martin Just) behandelte unter anderem Wilhelm Breitengraser und Caspar Othmayr; er befaßte sich mit Fragen der Rhetorisierung und Skandierung. Der Beitrag Cipriano de Rore und die italienisch-deutschen Musikbeziehungen (Prof. Dr. Dr. Lorenz Welker) untersuchte vor allem die Münchner Handschrift Chorbuch B und die Stellung der Vertonungen antiker Texte, ferner die Orientierung De Rores am Text und die Kunst, Stereotypien zu vermeiden. Das Problem 'humanistischer' Textdeklamation (Dr. Thomas Schmidt-Beste) wurde anhand der Textbehandlung in Kompositionen des 15. und frühen 16. Jahrhunderts erörtert, auch der Unterschied zwischen größerer Homorhythmie der Musik über humanistische Texte und Polyphonie, etwa sakraler Textbasis, wurde einbezogen. Der Vortrag Musik in der 
Enzyklopädik des Spätmittelalters und der frühen Neuzeit (Prof. Dr. Christel MeierStaubach) suchte die Komplexität 'humanistischer' Phänomene von einem Standpunkt außerhalb der Musikwissenschaft, dem der mittelalterlichen Philologie, aufzuzeigen. Die Rolle der Musik für den italienisch-französischen Humanismus des 15. Jahrhunderts (Prof. Dr. Reinhard Strohm) reflektierte den Gedanken der 'Wiedergeburt' in bezug auf die eloquentia, auch hinsichtlich der aemulatio (Nacheifern, auch Wettbewerb), einschließlich der Auswirkungen humanistischer Topoi auf den Bereich des Künstlertums, etwa der Vorstellung des Kunstwerkes, welches seinem Schöpfer Ruhm über den Tod hinaus einbringe. Der Beitrag Juan del Encina und die Bedeutung des Humanismus für die spanische Musik am Ende des 15. Jahrhunderts (Dr. Michael Zywietz) befaßte sich mit Isabellas Förderung des Humanismus und den Eigenarten von dessen spanischer, auch volkssprachlicher Variante, sodann auch mit dem italienischspanischen Austausch. Eine Interpretation von Walter Frye's Ave regina coelorum (Prof. Dr. David Fallows) zielte auf die Erscheinungsformen des frühen (musikalischen) Humanismus, im Hinblick auf die Bedeutung von Textstrukturen und bezüglich des Problems, daß eine geistliche Komposition im Kontext weltlicher Musik überliefert ist; Vergleiche mit Obrecht-Kompositionen dienten als Veranschaulichung. Das Glogauer Liederbuch und der schlesische Humanismus (Prof. Dr. Klaus Hortschansky) dokumentiert den kulturellen Austausch zwischen Schlesien und Italien; Eigentümlichkeiten, etwa das Zusammenbestehen älterer mittelalterlicher und neuerer humanistischer Lehrinhalte an der Krakauer Universität, Notationsbesonderheiten und die Beziehung des Liederbuches zur Humanistenode oder zum Chansonsatz dienten als Interpretaionsmuster. Der Beitrag Humanismus und Motivizität (Prof. Dr. Joshua Rifkin) hinterfragte einige vereinseitigende musikwissenschaftliche Vorstellungen, die sich mit dem Begriff des Humanismus verbinden; dazu zählt die perspektivische Ver-

kürzung, wie sich humanistisches Denken auf die Vertonung von Texten ausgewirkt habe. ('Motivizität' meint in diesem Zusammenhang alle Phänomene, die auf ein 
begrenztes Motivarsenal rückführbar sind; Imitation ist nur deren spezieller Fall.) Die Rezeption der Motette in Österreich unter dem Vorzeichen des Humanismus (Prof. Dr. Rudolf Flotzinger) untersuchte vor allem Huldigungsmotetten humanistischen Charakters, betonte den Umstand, daß die Ausrichtung am Text älter als das 'humanistische' Zeitalter sei und akzentuierte insbesondere die Malerei als Parallele zu musikalischen Entwicklungen im 15. Jahrhundert. In Humanismus und common sense Die Rolle der Musik in der englischen Fürstenspiegelliteratur des 16. Jahrhunderts (Dr. Dietrich Helms) ging es um die Spielart des englischen Humanismus, um die englische Antikenrezeption schon seit dem Mittelalter und um den Grad der Bedeutung der Musik als Erziehungsmittel. Das Referat Motettische Huldigungskompositionen des Humanismus und Späthumanismus (Prof. Dr. Klaus Wolfgang Niemöller) behandelte Huldigungskompositionen, Gegebenheiten des Symbolum und der Emblemata; ein Akzent fiel ebenfalls auf die späthumanistische Kultur am Prager Kaiserhof um 1600. Der Beitrag Orlando di Lasso und sein Aufenthalt in Antwerpen (Prof. Dr. Ignace Bossuyt) diskutierte den Einfluß humanistischer Kreise von Italienern in Antwerpen, aber auch Probleme chromatischer Musik und der Herkunft von Textvorlagen für Lassos weltliche Musiken. Die jesuitische Kritik am Gesangbuch der Böhmischen Brüder (Dr. Jaroslav Buzga) stellte die Traditionen der Beziehung zur Musik im böhmischen Raum vor, das Verhältnis der Hussiten zur Musik im Gegensatz zur humanistischen Kultur des Prager Hofes, die Theoretiker des Raumes Böhmen und den Einfluß, den das Zeitalter der Reformation auf dieses Gebiet nahm. Das Referat Höllenstrafe als Erziehungsmittel - Zu Hubert Waelrants 'Pater Abraham'-Vertonung im Kontext des 16. Jahrhunderts (PD Dr. Wolfgang Krebs) versuchte, anhand der Kriterien von Bildlichkeit oder Nichtbildlichkeit, Eigentlichkeit oder allegorischer Auffassung des Bibelwortes in der motettischen Konzeption, vor allem Waelrants und Lassos, zu Interpretationen im Sinne des Phänomens 'musikalischer Humanismus' zu gelangen. Der abschließende Beitrag über Ausdruck und Stilwandel am Beispiel der Huc me sidereo-Vertonungen (PD Dr. Rafael Köhler) untersuchte, vor allem am Beispiel Josquins, Textvertonungsprobleme, Bezüge zur älteren Isorhythmie, ebenso das Verhältnis von Satztechnik, Konstruktion und Expression bei Willaert und Lasso. Prof. Flotzingers Schlußwort, das Problem des Humanismus in der Musik sei letzen Endes nur auf dem Wege interdisziplinärer Betrachtungsweise zu bewältigen, unterstrich noch einmal eine der Intentionen der Tagung, die auch auf die Einbeziehung philosophischer und theologischer Neuzeitforschung zielte. 
Nicht unerwähnt sollte der Festvortrag von Prof. Dr. Ferdinand Seibt über Karl V. und das Ende des Mittelalters bleiben, denn er fand ebenso lebhaften Beifall wie die musikalische Umrahmung der Tagung durch die Münstersche Hofcantorey unter der Leitung von Garry Crighton, die Musik von Komponisten der Hofkapellen Isabellas, Ferdinands und Karls V. zu Gehör brachte.

Die Referate sollen in Aufsatzform binnen eines Jahres in einer eigenen Publikation erscheinen.

\section{Musik und Wissenschaft im Dialog. Eine Diskussionsveranstaltung des Musikwissenschaftlichen Instituts Frankfurt/Main im Rahmen von Wissenschaft erleben - Das Uni-Universum}

von Andreas Eichhorn

Am 22. November 1997 lud das Musikwissenschaftliche Institut der Johann Wolfgang Goethe-Universität Frankfurt/Main zu einer Gesprächsrunde in den Roten Saal des Poelzig-Casinos in Frankfurt ein, um zusammen mit Claus Kühnl (Komponist), Johann Werner Prein (Opern- und Konzertsänger), Dr. Eric Fiedler (Musiker und Musikwissenschaftler), Dr. Dietrich Berke (Cheflektor beim Bärenreiter Verlag Kassel) und Dr. Paul Fiebig (Redakteur beim Südwestdeutschen Rundfunk Baden) unter der Moderation von Prof. Dr. Adolf Nowak die Wechselbeziehungen zwischen Musikwissenschaft und öffentlichem Musikleben zu erörtern. Während der Komponist 
Claus Kühnl an zwei Ausschnitten eigener Werke demonstrierte, welches Inspirationspotential das von musikwissenschaftlicher Forschung erschlossene antike und mittelalterliche Musikdenken für seine eigene kompositorische Arbeit birgt, sahen Johann Werner Prein und Dr. Eric Fiedler im 'mündigen', d. h. musikwissenschaftlich selbständig arbeitenden Musiker eine Möglichkeit, die traditionelle Kluft zwischen Praktikern und Musikwissenschaftlern zu überbrücken. Dr. Dietrich Berke machte die Impulse deutlich, welche die von seiten der Musikverlage eingeläutete neue Ära der Gesamtausgaben nach dem Zweiten Weltkrieg auf die methodische Schärfung der musikwissenschaftlichen Textkritik ausübte. Dr. Paul Fiebig erläuterte am Beispiel einer Werkeinführung die Aufgabe des Redakteurs, musikwissenschaftliche Erkenntnisse hörergerecht aufzubereiten: Die Vermittlungsqualität des Rundfunks bestehe darin, der Musikwissenschaft 'Funken aufzusetzen'. An die einzelnen Beiträge schlossen sich Diskussionen mit reger Publikumsbeteiligung an.

\section{INTERNET-NACHRICHTEN}

Dr. Karlheinz Essl, Komponist. Geboren 1960 in Wien; Studium an der Wiener Musikhochschule (1981-87) in Komposition (Friedrich Cerha), elektro-akustische Musik (Dieter Kaufmann) und Kontrabaß (Heinrich Schneikart), ferner Studium der Musikwissenschaft und Kunstgeschichte an der Universität Wien (1979-89); 1989 Promotion mit einer Dissertation über Das Synthese-Denken bei Anton Webern. Kontrabassist in verschiedenen Kammermusik- und Jazz-Formationen. Auseinandersetzung mit mittelalterlicher Musik und deren Aufführungspraxis. Theoretische und kompositorische Aufarbeitung serieller Denkansätze. Untersuchungen zur Formalisierbarkeit musikalischer Prozesse (Computer Aided Composition) führen zur Entwicklung von Software-'Environments' für Algorithmische Komposition. Veröffentlichungen zur zeitgenössischen Kompositionstheorie. 1990-94 'composer in residence' bei den Darmstädter Ferienkursen für Neue Musik. 1992/93 PerformanceProjekt Partikel-Bewegungen mit Harald Naegeli, 1992/93 Kompositionsauftrag des 
IRCAM (Paris). Unterrichtet 'Computer Aided Composition' am Studio for Advanced Music and Media Technology des Bruckner-Konservatoriums in Linz. Gastvorlesungen in Graz, Toronto und Kopenhagen. Künstlerischer Betreuung der Konzertreihe Musik im Schömer-Haus. Neben Instrumentalwerken und Kompositionen mit Live-Elektronik auch Realtime-Kompositionen, Improvisationskonzepte, Klanginstallationen, InternetProjekte. Aufführungen bei zahlreichen internationalen Musikfestivals. [Weitere Informationen unter http://www.essl.at].

Karlheinz Essl veröffentlichte im Internet den Beitrag Kompositorische Konsequenzen des Radikalen Konstruktivismus. Abstract:

"Die epistemologische Einsicht, das die Wirklichkeit nicht bloße Abbildung einer objektiven Welt ist, sondern individuelle mentale Konstruktion zeitigt Konsequenzen für die kompositorische Praxis: das anvisierte Konzept der Offenheit ermöglicht dem Hörer, im aktiven Vorgang des Hörens seine persönliche Fassung des Werkes zu Ende zu komponieren - auf der Basis eines neuen, systemtheoretisch orientierten Materials, das der Komponist mit Hilfe des Computers erarbeitet und reflektiert."

Internet-Verbindung: http://www.essl.at/bibliogr/rad-konstr.html.

Deutschsprachige Mailinglisten. $\mathrm{Zu}$ Beginn des Jahres häuften sich technische Schwierigkeiten mit dem Server der Frankfurter Universität, die eine Weiterführung der ersten deutschsprachigen Mailingliste für Musik und Musikwissenschaft (MuWi@) als verantwortungslos erscheinen ließ: eine Zeitlang funktionierte die e-Mailadresse für das Listverwaltungsprogramm Majordomo nicht, und eine alte Teilnehmerdatei wurde verwendet.MuWi@ konnte nicht mehr konstruktiv genutzt werden; die Mailingliste wurde eingestellt. Schon ein paar Wochen nach dem Ende der MuWi@-Liste gingen zwei neue Mailinglisten zur Musik(wissenschaft) online: muwi-l (an der TU Berlin) 
und MuWiSpektrum (an der Uni Saarbrücken). Während MuWiSpektrum ein Forum für Musikwissenschaftler, Musikinformatiker, Musikpädagogen, Komponisten, Journalisten und Musikinteressierte sein möchte, soll muwi-1 sich besonders der Musikwissenschaft widmen. Für weitere Informationen siehe http://www.kgw.tuberlin.de/ fabri/forum/ bzw. http://www.coli.uni-sb.de/ zey/MuWiSpektrum.html.

Verzeichnis von Zeitschriften - Index von Komponisten. Sehr häufig werden die Seiten von Sonance (http://sonances.qc.ca/english.htm) in Kanada aktualisiert bzw. erweitert. Besonders die beiden großen Pfeiler des Informationsangebotes sind für Musikinteressierte und Musikwissenschaftler bedeutsam: ein Verzeichnis von Zeitschriften und eines zu Informationen über Komponisten, die auf dem Netz verfügbar sind. Letzteres (unter http://sonances.qc.ca/composers/contents.htm) beinhaltet Hyperlinks zu ca. 520 Komponisten und Komponistinnen; falls vorhanden, sind auch Links zu Unterrubriken, wie beispielsweise Werkverzeichnissen oder biographischen Informationen separat aufgelistet. Unter "International Survey of the Musical and Cultural Press" (siehe http://sonances.qc.ca/journals/pagerev.htm) werden etwa 160 musikwissenschafliche und musikbezogene Zeitschriften, sowie Kulturzeitschriften aufgeführt. Die jeweiligen Seiten zu einer Zeitschrift beinhalten die Kontaktadressen (meistens Vertriebsadresse), das Titelblatt und eine Reihe von Inhaltsverzeichnissen einiger Ausgaben: dies ist von Fall zu Fall sehr unterschiedlich, da Sonance vorwiegend die Aufbereitung zugesandter Informationen übernimmt und von der Mitarbeit einer großen Anzahl an Personen lebt. Alle namhafteren Zeitschriften der wissenschaftlichen Fachwelt finden sich hier, aber auch weniger bekannte Zeitungen und Journals. Die FZMw ist auch aufgeführt.

Englische Startseite: http://sonances.qc.ca/english.htm

Komponistenverzeichnis: $\underline{\text { http://sonances.qc.ca/composers/contents.htm }}$ 
Zeitschriftenverzeichnis: $\underline{\text { http://sonances.qc.ca/journals/pagerev.htm }}$

SEITE: 58

Electronic Musicological Review. Es handelt sich um eine zweisprachige (englisch und portugiesisch) Online-Zeitschrift aus Brasilien. Die Electronic Musicological Review erreicht man unter folgender URL: http://www.cce.ufpr.br/ rem/remi.html. Dort können aus dem bisher veröffentlichen Ausgaben, die teils portugiesischen, teils englischen Aufsätze im HTML-Format direkt gelesen werden. Pro Jahr erscheinen ein bis zwei Ausgaben. Bisherige Themen waren: Heitor Villa-Lobos: A Survey of his Guitar Music, A Nineteenth Century Brazilian Dulcimer Tablature, Os Modos e a Gênese Musical em Luis de Milan, Study on Logic Principles Applied to Musical Composition, Música Pré, Barroca Luso-Americana: O Grupo de Mogi das Cruzes, On Baroque Music and Brazil, Teoria Musical no Brasil: 1734-1854, A Obra Solística para Piano, Órgão e Cravo de José Penalva, A Obra Pianística do Padre José de Almeida Penalva - Carmen Célia Fregoneze, Breve Resenha das Contribuições de Schenker e Schoenberg para a Análise Musical, Schoenberg on Tonal Function, The Dangerous Issue of Modern Music in the Controversy Between Busoni and Pfitzner.

SEITE: 59

\section{DIE AUTOREN}

Michael Walter. Geboren 1958 in Gießen. Studium der Musikwissenschaft und Geschichte an den Universitäten Marburg und Gießen. 1985 Promotion. 1988-1989 als DFG-Stipendiat Kollegiat des Literatur- und Kommunikationswissenschaftlichen 
Graduiertenkollegs an der Universität Siegen. 1989-1993 Wissenschaftlicher Assistent am Institut für Sozialforschung der Universität Stuttgart. 1993 Habilitation für Musikgeschichte und Historische Verhaltensforschung an der Universität Stuttgart. Derzeit Hochschuldozent am Musikwissenschaftlichen Institut der Ruhr-Universität Bochum. Zahlreiche Publikationen zur Musikgeschichte des 18.-20. Jahrhunderts und des Mittelalters. Zuletzt erschienen: Hitler in der Oper. Deutsches Musikleben 1919-1945, Stuttgart/Weimar 1995, "Die Oper ist ein Irrenhaus". Sozialgeschichte der Oper im 19. Jahrhundert, Stuttgart/Weimar 1997; Musikkritik und Kanonisierung. Über E.T.A. Hoffmanns Rezension der 5. Sinfonie Beethovens, in: Musiktheorie 12 (1997), S. $255-265$.

Wolfgang Krebs. Geboren 1963 in Wiesen, Studium der Fächer Musikwissenschaft (v.a. bei Winfried Kirsch, Klaus Hortschansky, Lothar Hoffmann-Erbrecht), Geschichte und Germanistik an der Johann Wolfgang Goethe-Universität Frankfurt/Main; 1988 Magister Artium; 1993 Dr. phil. über das Thema Die lateinische Evangelien-Motette des 16. Jahrhunderts, 1996 Habilitation mit einer Arbeit Innere Dynamik und Energetik in Ernst Kurths Musiktheorie - Voraussetzungen, Grundzüge, analytische Perspektiven. Seit 1994 Lehrtätigkeit an der Frankfurter Universität; Vertretungen und Lehraufträge, u. a. an der Hochschule für Musik und Darstellende Kunst Frankfurt/Main.

Klaus Henning Oelmann. Geboren 1955 in Goslar/Harz, lebt seit 1985 in Norwegen. 1978 Magister artium an der Freien Universität Berlin, 1992 Promotion durch die Universität/ Gesamthochschule Kassel. Arbeit als Lehrer, Orchesterleiter, Bibliothekar, Musikforscher, Dozent für Musiktheorie und Musikgeschichte, zuletzt Ass. Prof. an der Hochschule in Tromsø, Fachbereich Musik, Tanz und Drama. Mitwirkung an der Edvard Grieg Gesamtausgabe. Forschungsschwerpunkte: Skizze und Kompositionsprozeß im Schaffen Edvard Griegs, Einfluß der Volksmusik auf die Harmonieentwicklung im späten 
19. Jahrhundert, Gehörbildungsdidaktik, Mentorpädagogik in der höheren Musikerziehung. Letzte Veröffentlichungen: Edvard Griegs Briefwechsel - Band 2 (Deutsche Hochschulschriften Bd. 1123), Egelsbach / St. Peter Port (1996): HänselHohenhausen / Knaresborough Ltd. Veiledning $i$ musikkpedagogutdanningen - Et innlegg [Die Bedeutung des Mentors im musikpädagogischen Studium - Ein Standpunkt], in: Arabesk - Kunstpedagogisk tidsskrift for musikk, dans og drama, Jg. 1 (1997), Heft 8, S. 36-41

Dokument erstellt am 25. März 1998

PD Dr. Wolfgang Krebs, Clemens Gresser 